\title{
Structure Activity Relationships of Quinoxalin-2-one Derivatives as Platelet-Derived Growth Factor- $\beta$ Receptor (PDGF $\beta$ R) Inhibitors, Derived from Molecular Modeling
}

\author{
Yoshikazu Mori ${ }^{a, d}$ Takatsugu Hirokawa, ${ }^{*, b}$ Katsuyuki AoKI,${ }^{a, d}$ Hisanori SATOMI, ${ }^{a}$ Shuichi TAKedA, ${ }^{a}$ \\ Masaki Aburada, ${ }^{c}$ and Ken-ichi MiYAmoto ${ }^{d}$ \\ ${ }^{a} R \&$ D Division, Tsumura \& Co.; 3586 Yoshiwara, Ami-machi, Inashiki-gun, Ibaraki 300-1192, Japan: ${ }^{b}$ Computational \\ Biology Research Center, National Institute of Advanced Industrial Science and Technology; 2-42 Aomi, Koto-ku, Tokyo \\ 135-0064, Japan: ' Research Institute of Pharmaceutical Sciences, Musashino University; 1-1-20 Shinmachi, Nishitokyo, \\ Tokyo 202-8585, Japan: 'Department of Clinical Pharmacy, Graduate School of Natural Science and Technology, \\ Kanazawa University; 13-1 Takaramachi, Kanazawa, Ishikawa 920-8641, Japan.
}

Received January 9, 2008; accepted February 21, 2008; published online March 3, 2008

\begin{abstract}
We previously reported a quinoxalin-2-one compound (Compound 1) that had inhibitory activity equivalent to existing platelet-derived growth factor- $\beta$ receptor (PDGF $\beta$ R) inhibitors. Lead optimization of Compound 1 to increase its activity and selectivity, using structural information regarding PDGF $\beta$ R-ligand interactions, is urgently needed. Here we present models of the PDGF $\beta$ R kinase domain complexed with quinoxalin-2-one derivatives. The models were constructed using comparative modeling, molecular dynamics (MD) and ligand docking. In particular, conformations derived from MD, and ligand binding site information presented by $\alpha$ spheres in the pre-docking processing, allowed us to identify optimal protein structures for docking of target ligands. By carrying out molecular modeling and MD of PDGF $\beta$ R in its inactive state, we obtained two structural models having good Compound 1 binding potentials. In order to distinguish the optimal candidate, we evaluated the structural activity relationships (SAR) between the ligand-binding free energies and inhibitory activity values $\left(\mathrm{IC}_{50}\right.$ values) for available quinoxalin-2-one derivatives. Consequently, a final model with a high SAR was identified. This model included a molecular interaction between the hydrophobic pocket behind the ATP binding site and the substitution region of the quinoxalin-2-one derivatives. These findings should prove useful in lead optimization of quinoxalin-2-one derivatives as PDGFb R inhibitors.
\end{abstract}

Key words platelet-derived growth factor- $\beta$ receptor; molecular modeling; ligand docking; molecular dynamics; quinoxalin-2one derivative; structure activity relationship

Platelet-Derived Growth Factor- $\beta$ Receptor (PDGF $\beta$ R) belongs to the receptor tyrosine kinase family, which also includes PDGF $\alpha$ R, Fms, Flt3, and Kit. ${ }^{1)}$ Members of this family are regarded as drug discovery targets for growth diseases such as various cancers, ${ }^{2)}$ antirestenosis, ${ }^{3-7)}$ atheroslerosis, $^{8,9)}$ fibrosis $^{10,11)}$ and nephritis. ${ }^{12)}$ A number of inhibitors, such as STI-571, ${ }^{13,14)}$ CT52923, ${ }^{15)}$ SU11248, ${ }^{16,17)}$ AGL2033/ $43^{18)}$ and RPR101511A, ${ }^{19)}$ have thus been found and developed. In particular, STI-571 is a very effective treatment for dermatofibrosarcoma protuberans (DFSP), chronic monomyelocytic leukemia (CMML) and so on. ${ }^{20}$ Through inhouse screening, we have found a new quinoxalin-2-one derivative, 7-[3-(cyclohexylmethyl)ureido]-3-\{1-methyl-1Hpyrrolo[2,3-b]pyridin-3-yl quinoxalin-2(1H)-one (Compound 1 in Table 1), that is essentially equivalent in effectiveness to existing inhibitors. Compound $\mathbf{1}$ was considered a lead compound, ${ }^{21)}$ and it was further optimized with the goal of improving its activity, leading to the development of 7-[3(pentyl)ureido]-3-\{1-methyl-1H-pyrrolo[2,3-b]pyridin-3yl quinoxalin-2(1H)-one (Compound 4 in Table 1). However, information regarding specific interactions between this compound and PDGF $\beta$ R awaits the determination of a 3D structure of PDGF $\beta$ R complexed with quinoxalin-2-one derivatives. Structural information is essential for rational inhibitor optimization. In this study, we used molecular modeling techniques to extract structural information regarding complexes formed by the PDGF $\beta$ R kinase domain and quinoxalin-2-one derivatives. We also evaluated structure activity relationships by utilizing $\mathrm{IC}_{50}$ values for in vitro inhi- bition of $\operatorname{PDGF} \beta$ R auto-phosphorylation in human aortic smooth muscle cells (AoSMC). Herein we report models of the complexes formed between $\operatorname{PDGF} \beta \mathrm{R}$ and quinoxalin-2one derivatives, and we evaluate structure activity relationships in light of these models.

\section{Experimental}

The molecular modeling of PDGF $\beta \mathrm{R}$ with quinoxalin-2-one derivatives involved four processes: comparative modeling, molecular dynamics (MD) simulation, ligand docking and SAR analysis. Receptor conformations optimized for ligand binding were selected from the ensemble of MD conformations before the ligand docking process was begun. Pre-docking processing of MD conformations, referred to as a "relaxed complex scheme," has been pioneered by McCammon's group. ${ }^{22-24)}$ In this work, we expand this process by evaluating the shapes of the ligand-binding pockets in conformations resulting from MD simulations. This pre-screening allows us to find optimized receptor conformations without having to carry out large scale docking simulations using all of the conformations produced by MD simulations. An experimental study related to the inhibitory activity of quinoxalin2-one derivatives to PDGF $\beta$ R has been described in detail previously. ${ }^{21)}$

Comparative Modeling The amino acid sequence of the PDGF $\beta \mathrm{R}$ kinase domain was obtained from the SWISS-PROT Database (ID: PGFRB_HUMAN [P09619]). Ordered and disordered regions in the PDGF $\beta$ $\mathrm{R}$ kinase domain were predicted using PSI-BLAST ${ }^{25)}$ and DISOPRED2.0. ${ }^{26}$ Before constructing a 3D model of PDGF $\beta$ R, a region predicted to be disordered was truncated and represented by a short pseudo-loop. The remaining region of $\operatorname{PDGF} \beta \mathrm{R}$ was built by comparative modeling based on the crystal structures of Flt3 (PDB-ID: 1RJB ${ }^{27}$ chain A), Lck (PDB-ID: $1 \mathrm{QPD}^{28)}$ chain A), and FGFR1 (PDB-ID: $1 \mathrm{FGI}^{29)}$ chain A) from the Protein Data Bank (PDB) ${ }^{30)}$ PSI-BLAST results indicated these proteins have a high sequence similarity with PDGF $\beta$ R, and they also have a similar inhibition profile with respect to quinoxalin-2-one derivatives. The PDGF $\beta$ R sequence was aligned with the three template protein sequences using ClustalW. ${ }^{31)}$ The $3 \mathrm{D}$ structure of PDGF $\beta$ R was constructed using the com- 
parative modeling approach incorporated in the program MODELLER6V2. ${ }^{32)}$ We generated 100 models for a sequence-templates alignment. All of the models were evaluated using the Verify3D program, ${ }^{33}$ which assigned each one a structure quality score. The highest-scoring model was chosen as the initial structure for an MD simulation.

MD Simulation An MD simulation was carried out using the AMBER8 package with the parm96 force field. The protein was surrounded with a $10 \AA$ layer of TIP3PBOX water molecules and its electrostatic charge was neutralized by adding counter ions, using the LeaP module of AMBER8 After minimization, heating and equilibration, the production MD phase was carried out at $300 \mathrm{~K}$ for $10 \mathrm{~ns}$, with a time step of $1 \mathrm{fs}$, using the constant volume and temperature (NVT) ensemble and the PME algorithm. All simulations were performed on a $8192 \mathrm{CPU}$ cores (4096 computing nodes) IBM BlueGene/L supercomputer at the Computational Biology Research Center (CBRC).

For trajectory analysis, $5000 \mathrm{MD}$ conformations, corresponding to each 1 -ps step of the final $5 \mathrm{~ns}$ trajectories, were saved using the ptraj command in AMBER8. The likeliest ligand-binding pocket within each of these conformations was detected using the SiteFinder module of MOE (Chemical Computing Group Inc.). The shape of each ligand-binding pocket was represented by small dummy atoms ( $\alpha$-spheres). To characterize the shapes of the ligand-binding pockets, the QuaSAR-descriptor program in MOE was used to compute the three principal moment of inertia (PMI) values $(x, y$ and $z$ components) for the cluster of small dummy atoms. The three pmi values of Compound 1 were also calculated in order to evaluate similarities between its shape and the ligand-binding pockets in the protein conformations produced by MD. The sum of the squared deviations of the three pmi values between the ligand-binding pocket and Compound $\mathbf{1}$ was employed as a shape similarity measure. This similarity measure was then used to rank the MD conformations, and the conformation having the most complementary binding pocket for Compound $\mathbf{1}$ was identified.

Structure Preparation for Docking The structures and activities of Compounds $1-7$, which have been well-studied experimentally, ${ }^{21)}$ used in this work are listed in Table 1. Initial coordinates of all compounds were constructed using the Molecular Builder module in MOE. Energy minimization of all compounds was performed using the OPLS-AA force field in the Conformational Search algorithm in the MacroModel program (Schrödinger Inc.). These minimized structures, with the exception of Compound 7, were employed as input structures for docking simulations. The lowest-energy structure of Compound 7 had a bend conformation involving the Octyl group, and it differed from the structure of Compounds $\mathbf{1 - 6}$. We manually selected a conformation with an extended Octyl group for Compound 7. The receptor structures with favorable shape similarity measures were prepared for docking simulations using the Protein Preparation Wizard Script within Maestro. This protein preparation procedure involves removal of waters, optimization of contacts by changing hydroxyl group orientations, flipping of Asn and Gln side chains, and selecting His tautomeric states, followed by constrained energy refinement using the OPLS-AA force field.

Table 1. Structure and Activity of Quinoxalin-2-one Derivatives ${ }^{21)}$

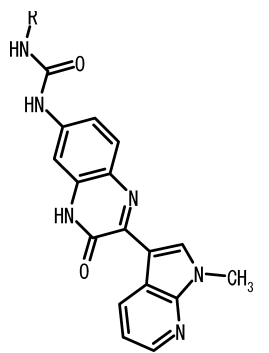

\begin{tabular}{ccc}
\hline \hline Compound & $\mathrm{R}$ & $\mathrm{IC}_{50}(\mu \mathrm{mol} / 1)^{a)}$ \\
\hline $\mathbf{1}$ & Cyclohexylmethyl & 0.030 \\
$\mathbf{2}$ & $n$-Propyl & 0.905 \\
$\mathbf{3}$ & $n$-Butyl & 0.020 \\
$\mathbf{4}$ & $n$-Pentyl & 0.007 \\
$\mathbf{5}$ & $n$-Hexyl & 0.010 \\
$\mathbf{6}$ & $n$-Heptyl & 0.050 \\
$\mathbf{7}$ & $n$-Octyl & 0.230 \\
\hline
\end{tabular}

a) $\mathrm{IC}_{50}$ values for the in vitro inhibition of $\operatorname{PDGF} \beta \mathrm{R}$ auto-phosphorylation in human AoSMC.
Docking Protocol Our approach to docking Compounds $\mathbf{1}-\mathbf{7}$ into the predicted PDGF $\beta$ R pockets utilized three main steps that take into account several levels of structural flexibility and scoring criteria: (1) molecular modeling of the Compound $\mathbf{1}-\mathrm{PDGF} \beta \mathrm{R}$ complex by docking Compound $\mathbf{1}$, considering both ligand and receptor flexibility, (2) rigid receptor docking of Compounds $1-7$ into the active site of PDGF $\beta$ R, which was defined using the Compound 1 complex obtained from the previous step and (3) re-scoring according to the calculated binding free energy.

In order to account for both ligand and receptor flexibility in the first step, the Glide 'Induced Fit Docking (IFD)' protocol ${ }^{34)}$ (Schrödinger Inc.) was utilized, followed by iteratively combining rigid receptor docking (Glide) and protein remodeling by side-chain searching and minimization (Prime) techniques. We generated 100 initial orientations of Compound $\mathbf{1}$ in a grid box defined by the center of the two key residues, Thr681 (Gatekeeper residue $)^{35)}$ and Phe845 (Asp-Phe-Gly motif) ${ }^{36)}$ using the Glide docking (standard precision (SP) mode). Next, the soften-potential docking options, which involve scaling the van der Waals radii by 0.25 for receptor and Compound 1 atoms and substitution of Tyr683 and Leu833 by alanine residues. We also introduced hydrogen-bonding constraints between the backbone NH of Cys684 and the backbone carbonyl of Glu682 in the hinge region of $\operatorname{PDGF} \beta \mathrm{R}$, because this hydrogen-bonding formation is highly conserved in almost all known complexes of kinases bound to ATP and to a wide variety of inhibitors. ${ }^{29,30,37-45)}$ In the protein remodeling stage, all residues within an $8.0 \AA$ radius of each initial docked Compound 1 were refined using Prime. Compound 1 was then re-docked into the refined receptor structure using Glide in the extra precision (XP) mode. All of the docked structures were then ranked according to an IFD score, which was based on the protein structure energy and the GlideScore (XP mode).

After modeling of the Compound 1-PDGF $\beta$ R complex using the IFD protocol, grid generation and rigid receptor docking of Compounds $1-7$ using Glide (XP mode) was carried out, using the hydrogen bonding constraint to connect a backbone $\mathrm{NH}$ and carbonyl pair in the hinge region, as well as other hydrogen bonding motifs observed in the Compound 1-PDGF $\beta$ $\mathrm{R}$ complex. Finally, the best orientation for each docked compound was rescored according to its binding free energy, $\Delta G_{\text {bind }}$, which was calculated using the Prime MM-GBSA module in Maestro ${ }^{46)}$ (Schrödinger Inc.).

\section{Results and Discussion}

Comparative Modeling The computer program DISOPRED2.0 was initially used to predict ordered and disordered regions in the initial $\operatorname{PDGF} \beta \mathrm{R}$ structure, and the kinase insert region $(700-792)$ was predicted to be disordered. The disordered region was then truncated and represented by a short pseudo-loop consisting of an Ala-Ala-Ala sequence.

Two earlier theoretical models of $\operatorname{PDGF} \beta \mathrm{R}$ have been reported. Both of these studies carried out comparative modeling based on the 3D structure of a kinase homologous to PDGF $\beta$ R. Gayathri et al. ${ }^{47)}$ used c-Src as a structural template, whereas comparative modeling of $\operatorname{PDGF} \beta \mathrm{R}$ using multiple templates (VEGFR2, FGFR1, c-Abl and insulin receptor kinase) was carried out by Böhmer et al. ${ }^{36)}$ These theoretical models allow one to evaluate the overall structural similarity of the templates to $\operatorname{PDGF} \beta$ R. However, the purpose of the present study is to accurately model quinoxaline2 -one derivatives bound to $\operatorname{PDGF} \beta \mathrm{R}$. Experimental information including an inhibition profile of Compound $\mathbf{1}$ with respect to various tyrosine kinases was therefore taken into account during template selection. According to the inhibition profile, along with direct radiometric assays for functional inhibition against a diverse panel of kinases using the KinaseProfiler service (Millipore Corporation, Billerica, MA, U.S.A.), Compound $\mathbf{1}$ also effectively inhibits FGFR3 (whose 3D structure is unknown), Flt3 (PDB-ID: 1RJB) and Lck (PDB-ID: 1QPD) activities. Therefore, we constructed a molecular model of PDGF $\beta$ R using FGFR1 (instead of FGFR3, PDB-ID: 1FGI), Flt3 and Lck as structural templates. Furthermore, since Compound $\mathbf{1}$ has been shown to 


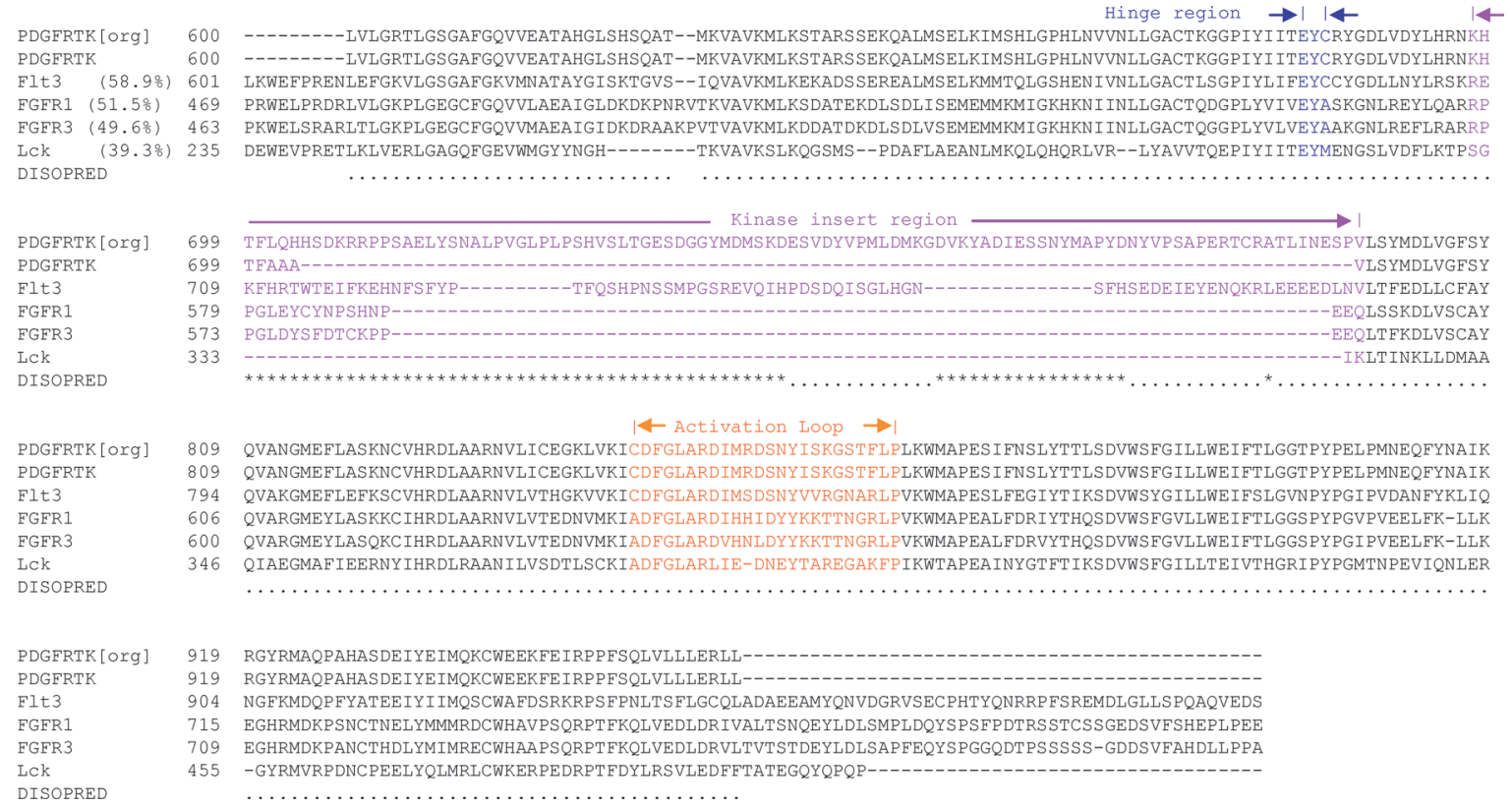

Fig. 1. The Protein Sequence Alignment of PDGF $\beta$ R and Four Tyrosine Kinases Flt3, FGFR1, FGFR3, Lck Used in Comparative Modeling Sequence alignment was performed using ClustalW. Asterisks $(*)$ represent disordered predictions and dots $(\cdot)$ prediction of order by DISOPRED. The hinge region, kinase insert region, and activation loop are in blue, magenta, and orange font, respectively.

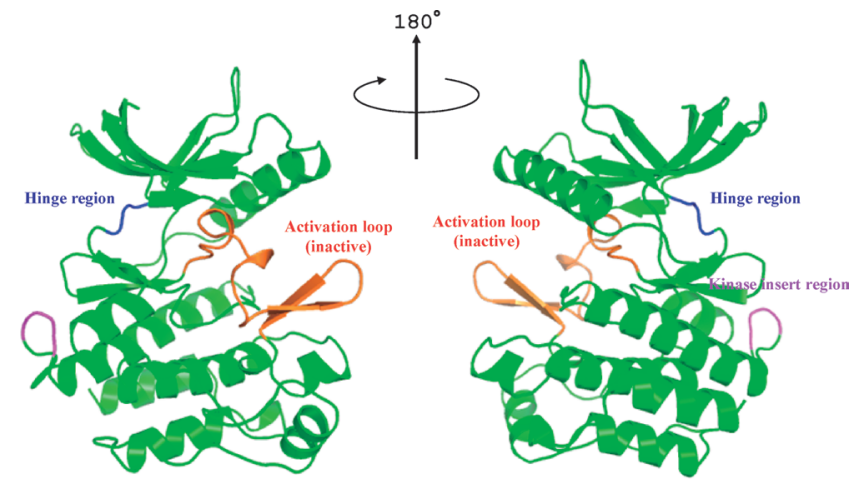

Fig. 2. Cartoon Representation of the PDGF $\beta$ R Kinase Model (Inactive Form)

Blue, magenta, and orange colors, respectively, indicate the hinge region, the kinase insert region replaced by Ala-Ala-Ala, and the activation loop.

inhibit auto-phosphorylation of $\operatorname{PDGF} \beta \mathrm{R}$, it apparently binds to and stabilizes an inactive form of PDGF $\beta$ R. In order to reflect this observation in comparative modeling, the PDGF $\beta$ R activation loop (residues 844-866) and its neighboring region (residues 606-614) were constructed using only Flt3 as a template, because its PDB structure corresponds to an inactive state. The sequence-structure alignment is shown in Fig. 1. The final model shown in Fig. 2 was selected from 100 conformations generated by MODELLER, and then selected based on their structure quality scores from the Verify3D protein structure evaluation program. The Verify3D score of the final model (0.99) was acceptable because it exceeded the incorrect fold score threshold score $(0.5)$.

Structure Refinement by MD The model selected after the comparative modeling step was then refined further using MD with explicit waters and the PME algorithm to generate pre-docking conformations of $\operatorname{PDGF} \beta \mathrm{R}$. Before beginning the $10 \mathrm{~ns}$ production phase, we performed an all-atom energy minimization, including $300 \mathrm{ps}$ for heating and $200 \mathrm{ps}$ for

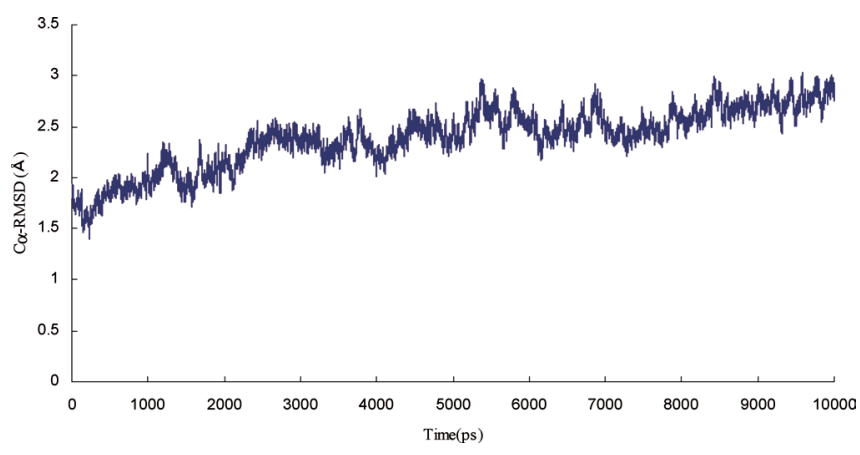

Fig. 3. The RMSDs of PDGF $\beta$ R (Blue Line) after a $10 \mathrm{~ns}$ MD Simulation with Explicit Waters at $300 \mathrm{~K}$

equilibration. Figure 3 shows the $\mathrm{C} \alpha$ root-mean-square deviations (RMSDs) from the initial structure after this production phase was carried out. The $\mathrm{C} \alpha$-RMSDs remained stable at $2.5-3.0 \AA$. Snapshots were taken every $1 \mathrm{ps}$ in the last $5 \mathrm{~ns}$ production phase (5000 snapshots), and these were then used for trajectory analysis. The ligand-binding pockets in the receptor conformations generated by this MD simulation were then characterized using a cluster of $\alpha$-spheres generated by the SiteFinder module in MOE. The ligand-binding pockets of the PDGF $\beta$ R structures included Glu682 and Cys684 (hinge region), Thr681 (Gatekeeper) and Phe845 (DFG motif), which can interact with the cluster of $\alpha$ spheres. As a result, 1673 configurations were found to have a potential ligand-binding pocket. In order to select the receptor configurations that are likeliest to bind Compound $\mathbf{1}$ from the 1673 candidates, we calculated the sum of squared deviations of three PMI values between the ligand-binding pocket and Compound $\mathbf{1}$ and sorted the structures accordingly. We used the five receptor configurations (Models 15) with the lowest sum of squared deviations as input structures for the next docking step.

Ligand Docking and SAR Analysis Induced fit docking 
Table 2. IFDScore of Models $1-5$

\begin{tabular}{cl}
\hline \hline Model & IFDScore $^{a)}$ \\
\hline 1 & -648.82 \\
2 & -543.03 \\
3 & -551.66 \\
4 & -536.39 \\
5 & -651.11
\end{tabular}

a) IFDScore $=$ GlideScore +0.05 PrimeEnergy

of Compound 1 was carried out starting with the five structures from the previous step, and using the soften-potential algorithm, alanine substitution and constrained hydrogenbonding interaction at the hinge region (as described in Experimentals) Table 2 reports the IFD scores for Compound 1 docked with the five receptor models. As seen in Table 2, Compound 1 bound to Models 1 and 5 had the lowest IFD scores. Models 1 and 5, showing predicted complexes of PDGF $\beta$ R with Compound 1, are illustrated in Fig. 4. Both models include interactions between backbone atoms ( $\mathrm{NH}$ of Cys684 and carbonyl of Glu682) in the hinge region of $\operatorname{PDGF} \beta$ R and the quinoxalin-2-one-group of Compound 1. However, the interaction profiles with a cyclohexyl-group in Compound 1 differed between the two models. The key residues of Model 1 that interacted with the cyclohexylgroup of Compound 1 were Leu652, Met655, Val665, Asn666, Leu667 and Ile679. In contrast, the key residues of Model 5 that interacted with the cyclohexyl-group in Compound 1 were Ile654, Met655, Leu658, Val664, Val665 and Ile679. In other words two different hydrophobic pockets in PDGF $\beta$ R could interact fruitfully with the cyclohexyl-group of Compound 1. Based on the information available at this point, i.e. the IFD score, it was not possible to select the single most likely binding mode for Compound 1. Therefore, rigid receptor docking was performed for Compounds $\mathbf{1}-\mathbf{7}$ using Models 1 and 5.

Experimental $\mathrm{IC}_{50}$ values are available for $\operatorname{PDGF} \beta$ R inhibition by a group of quinoxalin-2-one derivatives with a variety of substituted alkyl groups (Table 1). These values indicate that the alkyl group contributes to the interaction between quinoxalin-2-one derivatives and PDGF $\beta$ R. Thus the alternative models bound to Compound $\mathbf{1}$ could be evaluated by analyzing the rigid receptor docking of Compounds $\mathbf{1}-\mathbf{7}$. The binding free energies were calculated and correlated with $-\mathrm{pIC}_{50}$ values. Rigid docking of Compounds $\mathbf{1}-\mathbf{7}$ was carried out using Glide (XP mode). All of the docked conformations were then re-ranked according to their binding free energies $\left(\Delta G_{\text {bind }}\right)$, as calculated using the MM-GBSA program within Prime. The calculated binding free energies for Compounds $\mathbf{1}-\mathbf{7}$ docked into Models 1 and 5 are shown in Table 3. Figure 5 also shows the proposed binding modes for Compounds $1-7$ bound to Models 1 and 5. Comparison of binding free energies between Models 1 and 5 and correlation with $-\mathrm{pIC}_{50}$ are shown in Fig. 6. Compounds bound to Model 1 showed a correlation tendency between binding energies and $-\mathrm{pIC}_{50}$ values, whereas compounds bound to Model 5 showed a correlation tendency between binding energies and the lengths of the alkyl groups. Based on these results, Model 1 was chosen as the likeliest model for Compounds $1-7$ bound to PDGF $\beta$ R (Model 1: $R^{2}=0.77(0.77$ for Compounds 2-7), Model 5: $R^{2}=0.11$ (0.10 for Com-
Table 3. Binding Energy ( $\mathrm{kcal} / \mathrm{mol})$ Calculated by Prime MM-GBSA

\begin{tabular}{ccc}
\hline \multirow{2}{*}{ Compound } & \multicolumn{2}{c}{ Binding energy $(\mathrm{kcal} / \mathrm{mol})^{a)}$} \\
\cline { 2 - 3 } & Model 1 & Model 5 \\
\hline $\mathbf{1}$ & -79.52 & -77.68 \\
$\mathbf{2}$ & -75.71 & -66.54 \\
$\mathbf{3}$ & -79.02 & -73.26 \\
$\mathbf{4}$ & -82.23 & -74.26 \\
$\mathbf{5}$ & -83.61 & -74.47 \\
$\mathbf{6}$ & -76.76 & -78.78 \\
$\mathbf{7}$ & -75.81 & -79.47
\end{tabular}

a) Binding energy $=$ complex energy - receptor energy - ligand energy + ligand strain energy.

pounds $2-7)$ ).

The following was suggested as an interaction profile.

- The $\mathrm{C}=\mathrm{O}$ and $\mathrm{NH}$ of the quinoxalin-2-one ring form hydrogen bonds with the Cys684 (backbone NH) and Glu682 (backbone $\mathrm{C}=\mathrm{O}$ ) of the hinge region, respectively.

- The $\mathrm{C}=\mathrm{O}$ and $\mathrm{NH}$ of the urea moiety form hydrogen bonds with the Lys634 (side chain $\mathrm{NH}_{3}{ }^{+}$) and Thr681 (side chain $\mathrm{OH}$ ) of the hinge region, respectively.

- The $\mathrm{N}$ of the pyridine ring forms a hydrogen bond with Arg604 (side chain $\mathrm{NH}_{2}{ }^{+}$).

- The alkyl chains of Compounds $\mathbf{1}-\mathbf{6}$ interact with the hydrophobic pocket comprised of Leu652, Met655, Leu667 and Ile679, located in the depths of an ATP binding site.

Interestingly the interaction profile for the alkyl chain of Compound 7 was distinct from the others, and it resembled the profile of Model 5. This suggests that the hydrophobic pocket formed by Leu652, Met655, Leu667 and Ile679 could interact with alkyl group volumes up to the heptyl group degree. According to the binding energy of Compound $\mathbf{6}$ in both models, its profile resembles the interaction profile of Compound 7. However, it is difficult to compare the binding energy with different PDGF $\beta$ R models. To illustrate this, an interaction profile for Compound $\mathbf{1}$ bound to Model 1 is shown in Fig. 7. To understand the PDGF $\beta$ R selectivity for Compound $\mathbf{1}$ based on this interaction profile, we compared substitution of key residues indicated by the interaction profile with those of the following kinases: vascular endothelial growth factor receptor 2 (VEGFR2), epidermal growth factor receptor (EGFR), hepatocyte growth factor receptor (c-Met) and insulin growth factor 1 receptor (IGF-1R). Inhibitory potencies of Compound $\mathbf{1}$ for these kinases are about $3-4$ orders of magnitude lower $\left(\mathrm{IC}_{50}>10 \mu \mathrm{mol} / \mathrm{l}\right)$ than for PDGF $\beta$ $\mathrm{R}$. The interaction profile indicated that Leu652 of PDGF $\beta \mathrm{R}$ had a hydrophobic interaction with alkyl chains, and it corresponds to Gly1128 of c-Met, Ala739 of EGFR, and Ala1048 of IGF-1R. This suggested that the hydrophobic residue exchange at the Leu652 in PDGF $\beta$ R contributes significantly to the activity differences between $\operatorname{PDGF} \beta \mathrm{R}$ and these other kinases. In addition, Thr681 of PDGF $\beta$ R, which corresponds to Leu1157 of c-Met, Va1916 of VEGFR2, and Met1076 of IGF-1R, was hydrogen bonded with the urea moiety. The existence of a hydrophilic rather than a hydrophobic residue at this position in $\operatorname{PDGF} \beta \mathrm{R}$ may have influenced the selectivity of Compound $\mathbf{1}$. The model-derived information obtained through this study regarding PDGF $\beta \mathrm{R}$ interactions with quinoxalin-2-one derivatives may prove useful in future structure-based optimization studies. 

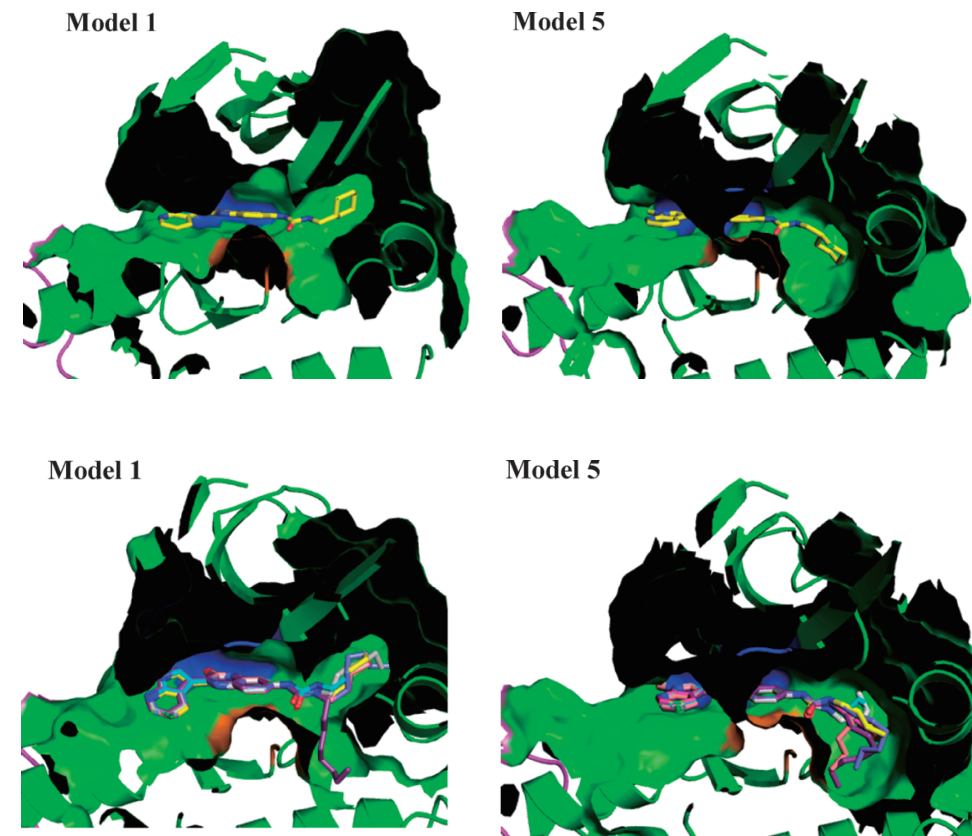

\section{mans}

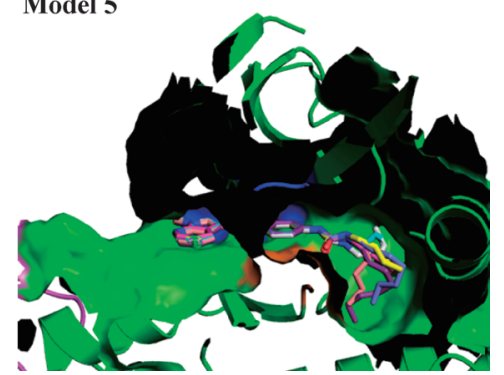

Fig. 4. Two Proposed Models for Compound 1 Binding to PDGF $\beta \mathrm{R}$, from Induced Fit Docking

Green surface indicates the Compound 1 binding pockets on Models 1 and 5. Blue, magenta, and orange surface colors indicate the hinge region, the kinase insert region replaced by Ala-Ala-Ala, and the activation loop, respectively. Compound $\mathbf{1}$ is shown as a stick model.
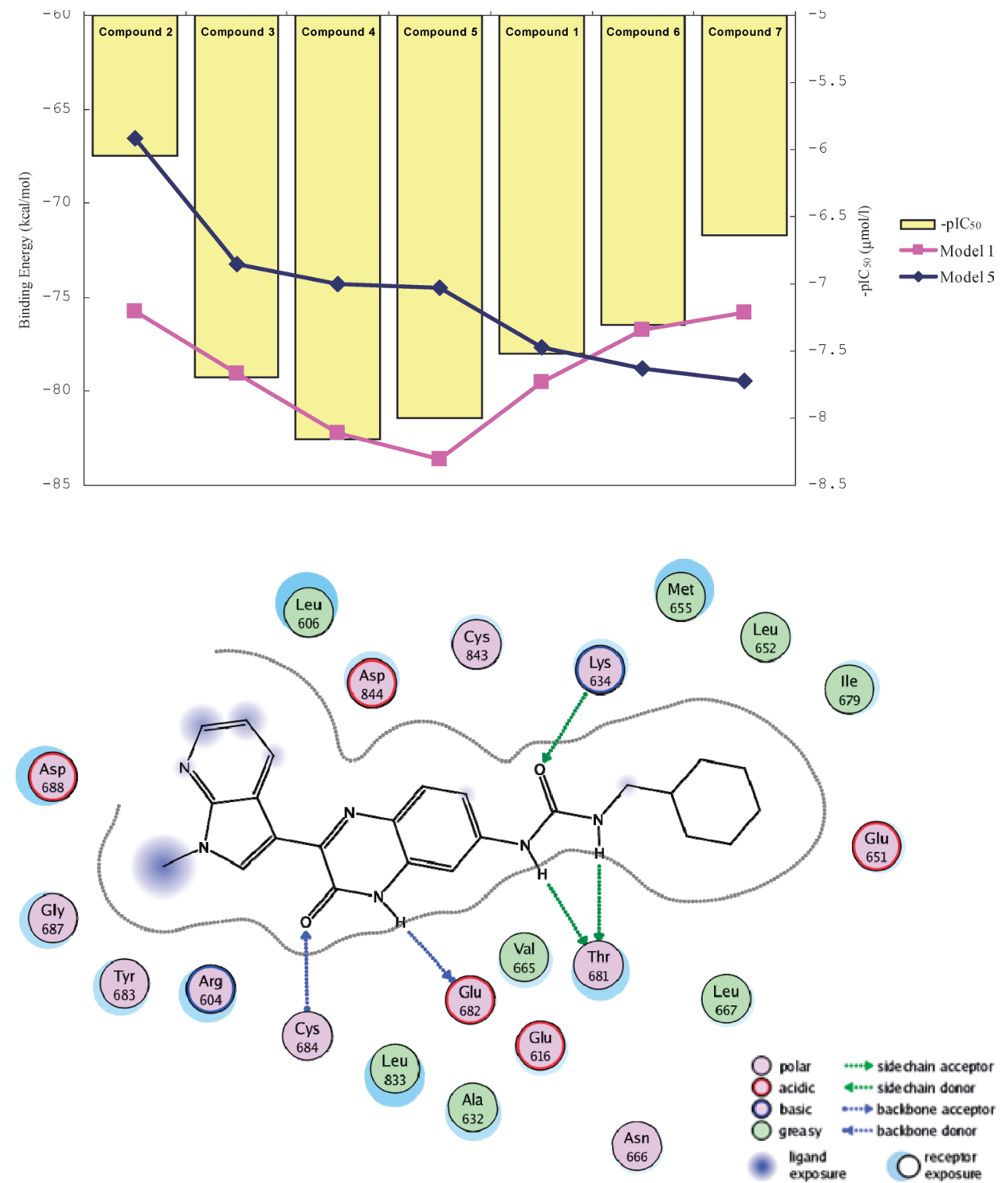

Fig. 6. Correlation $-\mathrm{pIC}_{50}$ Value $(\mu \mathrm{mol} / \mathrm{l})$ and Binding Energy $(\mathrm{kcal} / \mathrm{mol})$ of Calculated by Prime MM-GBSA

Pink and dark blue colors of line plots, respectively, indicate the binding energy of Compounds $1-7$ with Models 1 and 5 . Light yellow color of the bar plot indicates the $-\mathrm{pIC}_{50}$ values of Compounds $\mathbf{1}-\mathbf{7}$ for the inhibition of PDGF $\beta$ R auto-phosphorylation of human AoSMC in vitro.

Fig. 5. Two Proposed Models for Compounds $\mathbf{1}-\mathbf{7}$ Binding to PDGF $\beta$ R, from Rigid Docking Based on Models of the Com2; magenta, Compound 3; orange, Compound 4; white, Compound 5; slate, Compound 6; purple, Compound 7.

Fig. 7. Summary of the Interactions between Compound 1 and PDGF $\beta$ R

The 2-D representation was prepared using MOE. 


\section{Conclusions}

A pocket that could bind quinoxaline-2-one derivatives was present around an ATP binding site, and two distinct binding modes for quinoxaline-2-one derivatives have been suggested. Furthermore, the binding patterns revealed by modeling of alkyl chains of quinoxaline-2-one derivatives in the hydrophobic pocket within the ATP binding site showed correlations between binding scores and $\mathrm{IC}_{50}$ values. Therefore, interactions within this pocket apparently affect the activities of quinoxaline-2-one derivatives. The models of $\operatorname{PDGF} \beta \mathrm{R}$ complexed with quinoxaline-2-one derivatives were applied to structural prediction approaches to generate more active and selective PDGF $\beta$ RTK inhibitors. We will attempt to characterize the substitution of alkyl chains to other cyclic and branched structures that can interact with the hydrophobic pocket comprised of Leu652, Met655, Leu667 and Ile679 of PDGF $\beta$ R in future studies. Other future challenges will include utilizing this structural information and approach to investigate substitutions at regions other than the alkyl chains. In addition, we plan to carry out more detailed structural studies of various compounds bound to tyrosine kinase besides PDGF $\beta$ R.

\section{References}

1) Manning G., Whyte D. B., Martinez R., Hunter T., Sudarsanam S., Science, 298, 1912-1934 (2002).

2) Pietras K., Sjoblom T., Rubin K., Heldin C. H., Ostman A., Cancer Cell, 3, 439-443 (2003).

3) Grotendorst G. R., Seppa H. E., Kleinman H. K., Martin G. R., Proc. Natl. Acad. Sci. U.S.A., 78, 3669-3672 (1981).

4) Wilcox J. N., Smith K. M., Williams L. T., Schwartz S. M., Gordon D., J. Clin. Invest., 82, 1134-1143 (1988).

5) Ross R., Masuda J., Raines E. W., Gown A. M., Katsuda S., Sasahara M., Malden L. T., Masuko H., Sato H., Science, 248, 1009-1012 (1990).

6) Ferns G. A., Raines E. W., Sprugel K. H., Motani A. S., Reidy M. A., Ross R., Science, 253, 1129-1132 (1991).

7) Jawien A., Bowen-Pope D. F., Lindner V., Schwartz S. M., Clowes A. W., J. Clin. Invest., 89, 507-511 (1992).

8) Rutherford R. B., Ross R., J. Cell. Biol., 69, 196-203 (1976).

9) Ross R., Glomset J. A., N. Engl. J. Med., 295, 369-377 (1976).

10) Bonner J. C., Lindroos P. M., Rice A. B., Moomaw C. R., Morgan D. L., Am. J. Physiol., 274, L72-L80 (1998).

11) Friedman S. L., J. Biol. Chem., 275, 2247-2250 (2000).

12) Kurogi Y., Med. Res. Rev., 23, 15-31 (2003).

13) Kilic T., Alberta J. A., Zdunek P. R., Acar M., Iannarelli P., O’Reilly T., Buchdunger E., Black P. M., Stiles C. D., Cancer Res., 60, 51435150 (2000).

14) Buchdunger E., Cioffi C. L., Law N., Stover D., Ohno-Jones S., Druker B. J., Lydon N. B., J. Pharmacol. Exp. Ther., 295, 139-145 (2000).

15) Yu J. C., Lokker N. A., Hollenbach S., Apatira M., Li J., Betz A., Sedlock D., Oda S., Nomoto Y., Matsuno K., Ide S., Tsukuda E., Giese N. A., J. Pharmacol. Exp. Ther., 298, 1172-1178 (2001).

16) Abrams T. J., Lee L. B., Murray L. J., Pryer N. K., Cherrington J. M., Mol. Cancer Ther, 2, 471-478 (2003).

17) Mendel D. B., Laird A. D., Xin X., Louie S. G., Christensen J. G., Li G., Schreck R. E., Abrams T. J., Ngai T. J., Lee L. B., Murray L. J., Carver J., Chan E., Moss K. G., Haznedar J. O., Sukbuntherng J., Blake R. A., Sun L., Tang C., Miller T., Shirazian S., McMahon G., Cherrington J. M., Clin. Cancer Res., 9, 327-337 (2003).

18) Gazit A., Yee K., Uecker A., Bohmer F. D., Sjoblom T., Ostman A., Waltenberger J., Golomb G., Banai S., Heinrich M. C., Levitzki A., Bioorg. Med. Chem., 11, 2007-2018 (2003).
19) Bilder G., Wentz T., Leadley R., Amin D., Byan L., O’Conner B., Needle S., Galczenski H., Bostwick J., Kasiewski C., Myers M., Spada A., Merkel L., Ly C., Persons P., Page K., Perrone M., Dunwiddie C., Circulation, 99, 3292-3299 (1999).

20) Magnusson M. K., Meade K. E., Nakamura R., Barrett J., Dunbar C. E., Blood, 100, 1088-1091 (2002).

21) Aoki K., Obata T., Yamazaki Y., Mori Y., Hirokawa H., Koseki J., Hattori T., Niitsu K., Takeda S., Aburada M., Miyamoto K., Chem. Pharm. Bull., 55, 255-267 (2007).

22) Lin J. H., Perryman A. L., Schames J. R., McCammon J. A., J. Am. Chem. Soc., 124, 5632-5633 (2002)

23) Lin J. H., Perryman A. L., Schames J. R., McCammon J. A., Biopolymers, 68, 47-62 (2003)

24) Schames J. R., Henchman R. H., Siegel J. S., Sotriffer C. A., Ni H., McCammon J. A., J. Med. Chem., 47, 1879-1881 (2004).

25) Altschul S. F., Madden T. L., Schaffer A. A., Zhang J., Zhang Z., Miller W., Lipman D. J., Nucleic Acids Res., 25, 3389-3402 (1997).

26) Ward J. J., McGuffin L. J., Bryson K., Buxton B. F., Jones D. T., Bioinformatics, 20, 2138-2139 (2004).

27) Griffith J., Black J., Faerman C., Swenson L., Wynn M., Lu F., Lippke J., Saxena K., Mol. Cell, 13, 169-178 (2004).

28) Zhu X., Kim J. L., Newcomb J. R., Rose P. E., Stover D. R., Toledo L. M., Zhao H., Morgenstern K. A., Structure, 7, 651-661 (1999).

29) Mohammadi M., McMahon G., Sun L., Tang C., Hirth P., Yeh B. K., Hubbard S. R., Schlessinger J., Science, 276, 955-960 (1997).

30) Berman H. M., Westbrook J., Feng Z., Gilliland G., Bhat T. N., Weissig H., Shindyalov I. N., Bourne P. E., Nucleic Acids Res., 28, 235 242 (2000).

31) Thompson J. D., Higgins D. G., Gibson T. J., Nucleic Acids Res., 22, 4673-4680 (1994).

32) Sali A., Blundell T. L., J. Mol. Biol., 234, 779-815 (1993).

33) Luthy R., Bowie J. U., Eisenberg D., Nature (London), 356, 83-85 (1992).

34) Sherman W., Day T., Jacobson M. P., Friesner R. A., Farid R., J. Med Chem., 49, 534-553 (2006).

35) Noble M. E., Endicott J. A., Johnson L. N., Science, 303, 1800-1805 (2004).

36) Bohmer F. D., Karagyozov L., Uecker A., Serve H., Botzki A., Mahboobi S., Dove S., J. Biol. Chem., 278, 5148-5155 (2003).

37) Nagar B., Hantschel O., Young M. A., Scheffzek K., Veach D., Bornmann W., Clarkson B., Superti-Furga G., Kuriyan J., Cell, 112, 859871 (2003).

38) Pautsch A., Zoephel A., Ahorn H., Spevak W., Hauptmann R., Nar H., Structure, 9, 955-965 (2001).

39) Schindler T., Sicheri F., Pico A., Gazit A., Levitzki A., Kuriyan J., Mol. Cell, 3, 639-648 (1999).

40) Nowakowski J., Cronin C. N., McRee D. E., Knuth M. W., Nelson C. G., Pavletich N. P., Rogers J., Sang B. C., Scheibe D. N., Swanson R. V., Thompson D. A., Structure, 10, 1659-1667 (2002).

41) Wybenga-Groot L. E., Baskin B., Ong S. H., Tong J., Pawson T., Sicheri F., Cell, 106, 745-757 (2001).

42) Schiering N., Knapp S., Marconi M., Flocco M. M., Cui J., Perego R., Rusconi L., Cristiani C., Proc. Natl. Acad. Sci. U.S.A., 100, 12654 12659 (2003)

43) Witucki L. A., Huang X., Shah K., Liu Y., Kyin S., Eck M. J., Shokat K. M., Chem. Biol., 9, 25-33 (2002).

44) Mol C. D., Lim K. B., Sridhar V., Zou H., Chien E. Y., Sang B. C., Nowakowski J., Kassel D. B., Cronin C. N., McRee D. E., J. Biol. Chem., 278, 31461-31464 (2003).

45) Mol C. D., Dougan D. R., Schneider T. R., Skene R. J., Kraus M. L., Scheibe D. N., Snell G. P., Zou H., Sang B. C., Wilson K. P., J. Biol. Chem., 279, 31655-31663 (2004).

46) Lyne P. D., Lamb M. L., Saeh J. C., J. Med. Chem., 49, 4805-4808 (2006).

47) Gayathri K., Muthuvel A., Protein Data Bank, ID: 1LWP (theoretical model). 